\title{
Multiplicity of magnetic domain states in circular elements probed by photoemission electron microscopy
}

\author{
C. A. F. Vaz, ${ }^{1}$ M. Kläui $,{ }^{1}, *$ L. J. Heyderman, ${ }^{2}$ C. David,${ }^{2}$ F. Nolting,${ }^{3}$ and J. A. C. Bland ${ }^{1, \dagger}$ \\ ${ }^{1}$ Cavendish Laboratory, University of Cambridge, Cambridge, CB3 OHE, United Kingdom \\ ${ }^{2}$ Laboratory for Micro- and Nanotechnology, Paul Scherrer Institut, Villigen PSI, Switzerland \\ ${ }^{3}$ Swiss Light Source, Paul Scherrer Institut, Villigen PSI, Switzerland
}

(Received 26 May 2005; revised manuscript received 20 July 2005; published 19 December 2005)

\begin{abstract}
A systematic study of the remanent magnetic states in $\mathrm{Co}$ and $\mathrm{Ni}_{80} \mathrm{Fe}_{20}$ disks $1.65 \mu \mathrm{m}$ in diameter with varying film thickness $(5-38 \mathrm{~nm})$ imaged by a noninvasive imaging technique (namely, photoemission electron microscopy) is presented. The magnetic configurations observed range from the vortex state, to other well defined multidomain states, namely a state with two vortex cores ("diamond" state) and a high remanence "triangle" state, which are mapped according to material and thickness range. In most instances, more than one magnetic state coexist in different disks of the same array, subjected to the same field history. These observations are correlated with magneto-optical Kerr effect measurements and micromagnetic simulations to show that the observed magnetic metastable states are stabilized by defects or attained in the nucleation process following the removal of the applied magnetic field.
\end{abstract}

DOI: 10.1103/PhysRevB.72.224426 PACS number(s): 75.60. $-\mathrm{d}$, 75.75.+a, 75.25.+z, 75.70.Kw

\section{INTRODUCTION}

Although the groundwork for the understanding of micromagnetism was laid down several decades ago, ${ }^{1-7}$ a better appreciation is now emerging of the importance of the different energy terms to the switching behavior of mesoscopic elements and to the magnetic states that are, thenceforth, attainable at remanence. ${ }^{8-14} \mathrm{~A}$ critical parameter in small elements is the physical shape, since the long range dipolar interaction acts in such a way as to reduce the magnetostatic self-energy by favoring flux-closure configurations. ${ }^{13-17}$ The effect of the magnetocrystalline anisotropy is usually that of introducing local domains pointing along the easy magnetization axes, ${ }^{13,14,18}$ in another instance, it has been shown that for very small polycrystalline elements, the small number of crystallites present result in a "residual" magnetocrystalline anisotropy which may affect the switching process and the direction of magnetization in single domain particles at remanence. ${ }^{19-21}$ These conflicting energy contributions lead to a rich magnetic behavior that is a function of the geometrical parameters of the element and of the magnetic material employed, and which can be harnessed both for applications, such as memory cell elements for MRAM and sensors, ${ }^{22-24}$ as well as for the study of fundamental issues in the static and dynamic behavior of submicrometer magnetic systems, for example current-induced switching of the magnetization, ${ }^{25-28}$ where optimization of the domain wall structure is critical for efficient switching. ${ }^{23,29-35}$ In the latter example, it is observed that the high density currents induce distortions in the magnetic domain wall ${ }^{34,36}$ and in extreme cases lead to magnetic states which are not the lowest energy configurations. ${ }^{37,38}$ Although in this case the metastable states are attained though complex magnetization dynamics, ${ }^{39-41}$ similar processes occur in quasistatic situations when the local magnetization is pinned at defects or other pinning centers during magnetization reversal, preventing the system from attaining the ground state. In general, these metastable magnetic configurations may be accessible depending on the particular path followed by the magnetization, and may be stable against thermal or other external perturbations depending on the energy barrier that separates it from the ground state. Therefore, the study of such metastable states allows one to better understand the energetics of small elements and the magnetization dynamics involved in particular field paths. Here we report a systematic study of the remanent magnetic states in $\mathrm{Co}$ and $\mathrm{NiFe}$ disks imaged by photoemission electron microscopy (PEEM). While one expects that for these high symmetry elements the vortex state constitutes the state of lowest energy, we show that for many systems (as defined by a particular material, crystalline phase and thickness), more than one magnetic state coexist in different disks of the same array. Our results illustrate the importance of the different energy terms in determining the equilibrium state, and also the importance of the details of the switching processes vis-à-vis the magnetic defects that act as pinning sites, or nucleation sites for switching of the magnetization.

\section{SAMPLE GROWTH AND EXPERIMENTAL TECHNIQUES}

Epitaxial fcc $\mathrm{Co}(001)$ and polycrystalline (hcp) Co and (fcc) $\mathrm{Ni}_{80} \mathrm{Fe}_{20}$ films were deposited on a PMMA (polymethylmethacrylate) masked $\mathrm{Si}(001)$ substrate, followed by a liftoff step to remove the unwanted magnetic material. The resist mask, consisting of a negative of the disk pattern, was first created by $e$-beam exposure of the PMMA resist layer, spin-coated on the $\mathrm{Si}(001)$ substrate. ${ }^{42,43}$ The patterned elements considered here consist of disks with the outer diameter set to $1.65 \mu \mathrm{m}$ and with a separation distance of $3.5 \mu \mathrm{m}$ such that the interaction between the disks is negligible (this is the case when the distance separating the elements is larger than the physical dimension of the element ${ }^{44-46}$ ). These dimensions were confirmed by scanning electron 
microscopy (SEM) and can be used as a reference lengthscale for the PEEM images presented in this study. The epitaxial fcc Co film was obtained by deposition of a thick $\mathrm{Cu}$ buffer layer onto the HF etched Si substrate, ${ }^{42,47-50}$ while the polycrystalline samples (with no overall magnetic anisotropy) were obtained by deposition of the metal film directly onto the naturally oxidized $\mathrm{Si}$ surface. ${ }^{51}$ Two epitaxial fcc Co set of structures were grown with nominal sample structure of the form $\mathrm{Al}(0.5 \mathrm{~nm}) /$ $\mathrm{Cu}(1.3 \mathrm{~nm}) / \mathrm{fcc} \mathrm{Co}(29 \mathrm{~nm}) / \mathrm{Cu}(75 \mathrm{~nm}) / \mathrm{Si}(001)$ and $\mathrm{Al}(0.5 \mathrm{~nm}) / \mathrm{fcc} \mathrm{Co}(16 \mathrm{~nm}) / \mathrm{Cu}(35 \mathrm{~nm}) / \mathrm{Si}(001)$ while a range of thicknesses for the polycrystalline $\mathrm{Co}$ and $\mathrm{NiFe}$ structures were grown, with nominal sample structure of the form $\mathrm{Al}(0.5 \mathrm{~nm}) / \mathrm{Co}, \mathrm{Ni}_{80} \mathrm{Fe}_{20} / \mathrm{SiO}_{x} / \mathrm{Si}(001)$. The metal deposition was done in an ultrahigh vacuum MBE system with a base pressure $\sim 3 \times 10^{-10}$ mbar, and during deposition of the $\mathrm{Cu}$ buffer layer the pressure increased to $\sim 3$ $\times 10^{-9}$ mbar (evaporation rate of $\sim 0.5 \mathrm{~nm} / \mathrm{min}$ ) while for the other layers it remained below $\sim 8 \times 10^{-10}$ mbar (evaporation rates $\sim 0.2 \mathrm{~nm} / \mathrm{min}$ ). The deposition of the magnetic layers (Co and $\mathrm{NiFe}$ ) was performed with the sample at normal incidence with respect to the sources. The Al capping layer was chosen as a protective layer against oxidation and also because it has a large electron mean free path for the photoemitted electrons detected by PEEM as compared with other materials also employed as protective layers ( $\mathrm{Au}$ or Cr). After growth, the samples were removed for ex situ magnetic characterization. The $M-H$ hysteresis loops were measured using magneto-optic Kerr effect (MOKE) magnetometry on large arrays of identical disks, while the magnetic imaging was performed on smaller arrays of 25 elements using photoemission electron microscopy, ${ }^{52-56}$ conducted at the SIM beamline at the Swiss Light Source (Switzerland). In this technique, changes in the photoemission intensity at the absorption edges of the material element with the relative orientation between the (circular) light polarization and magnetization is used to obtain directly an image of the magnetization distribution in the magnetic system (the magnetic contrast is along the direction of the light polarization). It is a noninvasive technique in that no magnetic field interaction is present [unlike magnetic force microscopy (MFM)] and therefore is particularly well suited for magnetic imaging of soft magnetic elements (the stray field from the magnetic lenses is below 1 Oe for both the in-plane and out of plane components). All measurements reported here were performed at room temperature and all PEEM images were obtained at remanence, under no applied magnetic field, after saturation in a magnetic field of $4 \mathrm{kOe}$, well above the saturation field of all the structures studied here. The procedure for obtaining the magnetic images was to calculate the ratio between the photoemitted electron intensities with right and left circularly polarized light, after correcting for sample drift. This is sufficient for a qualitative interpretation of the magnetic domain configuration.

\section{EXPERIMENTAL RESULTS}

The $M-H$ curves measured for different thicknesses of the polycrystalline Co disks show a variation consistent with

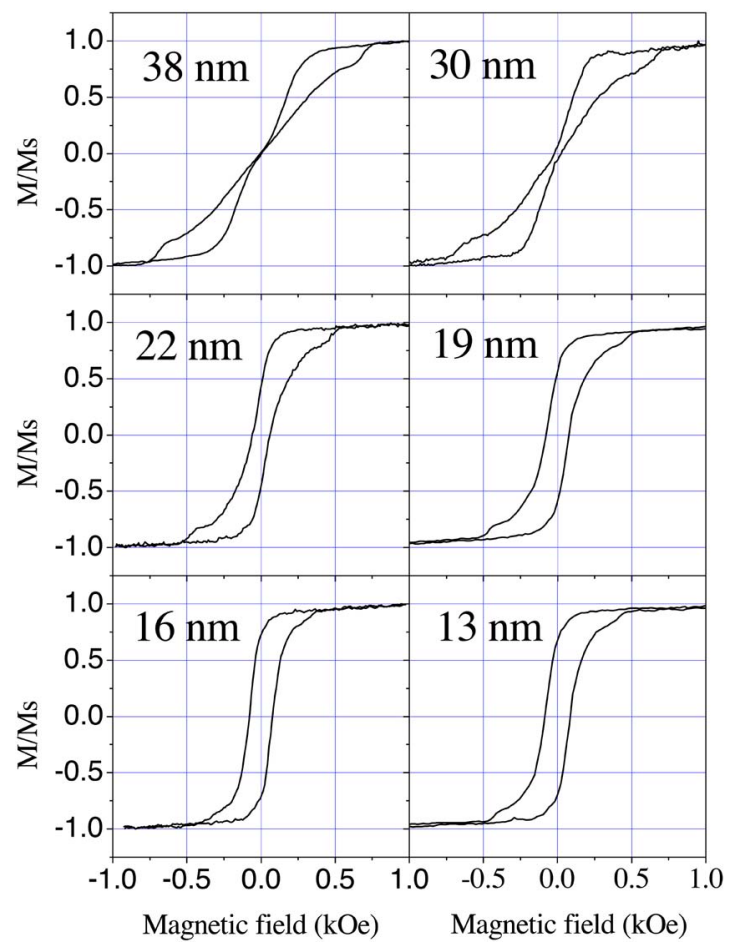

FIG. 1. (Color online) $M-H$ characteristics for different thicknesses of polycrystalline Co disks.

previous reports, ${ }^{57}$ where the magnetization switches in a two step process for all the thickness range, Fig. 1. The first drop in magnetization, when decreasing the field from saturation, corresponds to the nucleation of a vortex during the relaxation process, and the second jump at high reverse fields correspond to the annihilation of the vortex core in the transition to the saturated state. For example, for the $38 \mathrm{~nm}$ polycrystalline Co disks shown in Fig. 1, the transition from the saturated state to the vortex state starts at $\sim 350$ Oe, while the vortex annihilation occurs at around -650 Oe for the same branch of the $M-H$ curve (we note that the magnetic field at which both these transitions occur decrease in amplitude with decreasing disk thickness). We see that for thicker Co disks the remanence is close to zero and increases as the disk thickness decreases. This suggests that for the thinner films, not all disks fall into the vortex state (which has zero remanence); similarly, the coercivity is also seen to increase as the Co thickness decreases. These results are summarized in Fig. 2 which shows the variation of the remanent magnetization and coercivity as a function of thickness for the wide spaced polycrystalline Co disks. The trend towards zero remanence with increasing thickness is expected, since the magnetostatic energy of in-plane magnetized states increases quadratically with the element thickness. The behavior of the polycrystalline $\mathrm{NiFe}$ disks is similar, with a small reduction in coercivity from the $10 \mathrm{~nm}$ to the $5 \mathrm{~nm}$ disks.

The explanation for the differences in remanence as a function of thickness is provided by the PEEM results. For thick disks $(30-38 \mathrm{~nm})$ the remanence and coercive field are small, suggesting that most of the disks fall into the vortex state, as mentioned above. This is shown in Fig. 3 for the $38 \mathrm{~nm}$ Co disks, where most disks are observed to have 


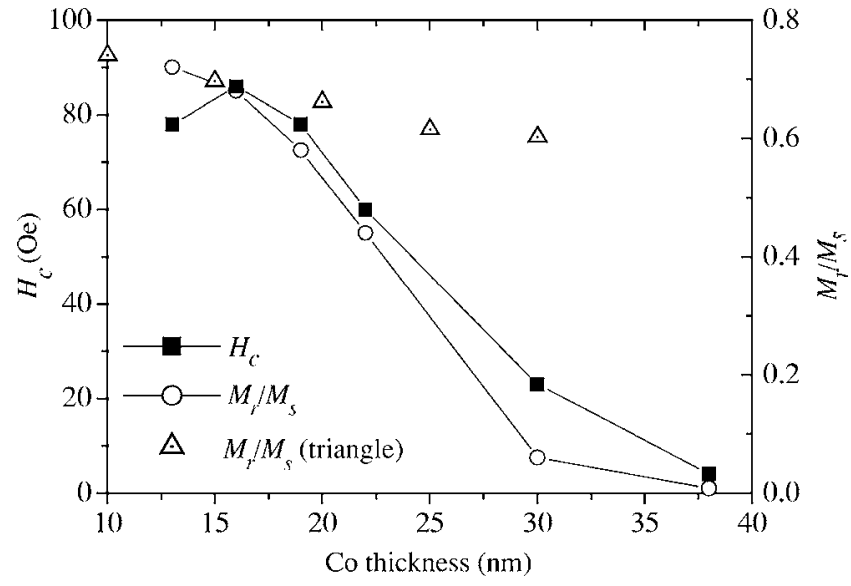

FIG. 2. Variation of the remanent magnetization $M_{r} / M_{s}$ (right axis) and coercivity $H_{c}$ (left axis) as a function of thickness for the polycrystalline Co disks. The triangles correspond to the remanence of the triangle state as determined from the results of micromagnetic simulations.

fallen into the vortex state (characterized by a black and white quadrant along the direction of the light polarization and separated by two grey quadrants along the perpendicular direction; both senses of magnetization rotation are present), with the exception of a single disk (of the array of 24 visible, indicated by an arrow in Fig. 3) which has fallen into a state consisting of three domains: two outer domains (white contrast) pointing along one direction and the middle one (black contrast) pointing in the opposite direction. This state will be referred to as the "diamond" state and it will be shown that it prevails for certain Co thicknesses and is usually present throughout the disk arrays (also for the $\mathrm{NiFe}$ and fcc Co disks).

For thinner disks the coercivities and remanence are very large, which indicate that a significant energy barrier prevents most disks from falling into the vortex state (which is

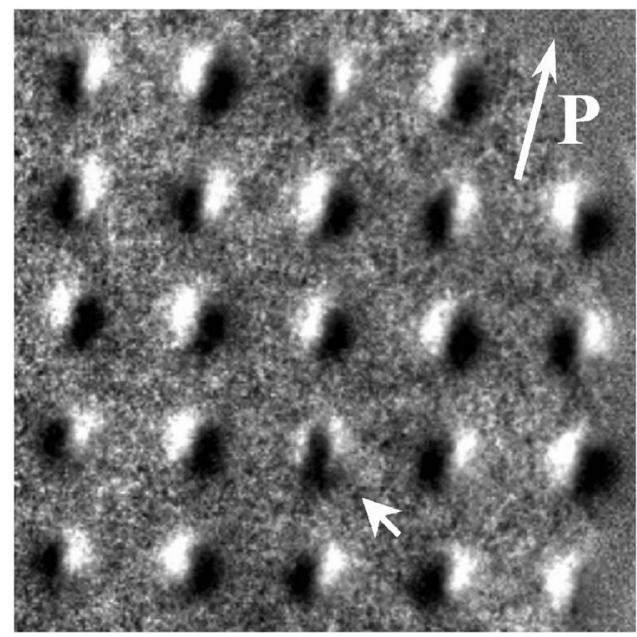

FIG. 3. PEEM image of the $38 \mathrm{~nm}$ Co polycrystalline disks in the remanent state after saturation (magnetic field applied along the direction of light polarization $\mathbf{P}$ ). All disks are in the vortex state except the one indicated with an arrow, which is in the diamond state.

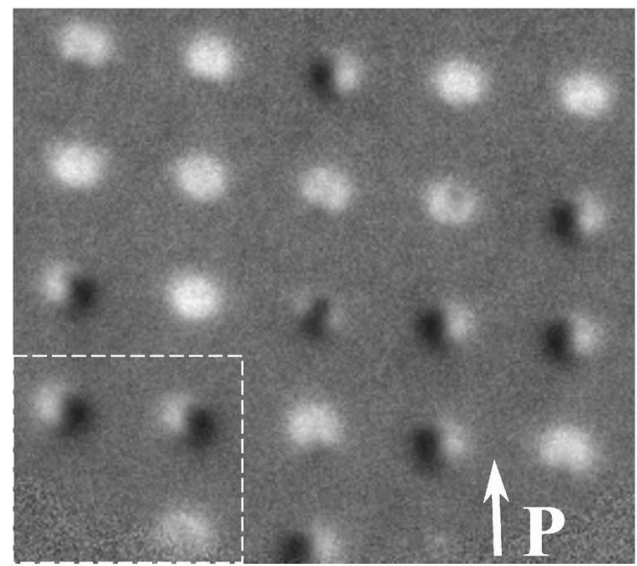

(a)

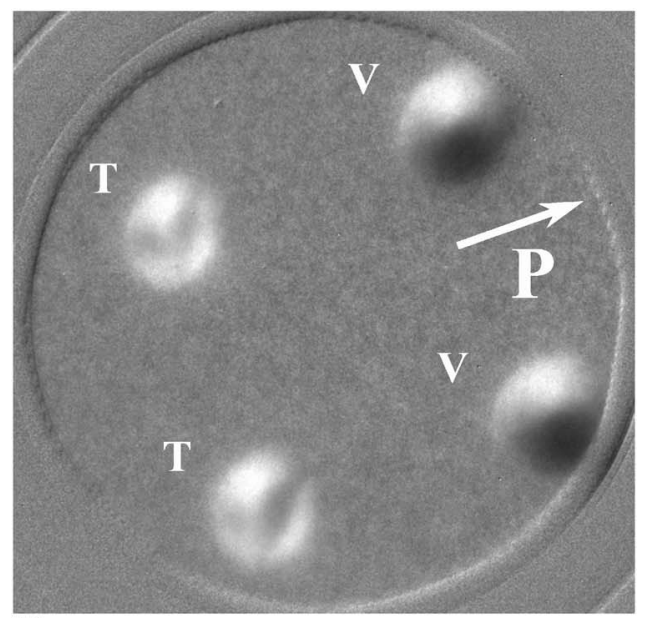

(b)

FIG. 4. PEEM images of the $19 \mathrm{~nm}$ Co polycrystalline disks in the remanent state after saturation (magnetic field applied along the direction of the light polarization $\mathbf{P}$ ). The magnetic states of the disks on the high resolution image (marked on the top image) are identified as triangle $(T)$ and vortex $(V)$ states (note the clockwise rotation of the image, which is due to the magnetic lenses of the microscope).

expected to be the ground state configuration for the disks considered here, see Sec. IV), and remaining instead in a state of overall nonzero magnetic moment. For instance, the PEEM images of the $19 \mathrm{~nm}$ polycrystalline Co disks show many of the disks in a state resembling the saturated state, which on closer inspection turns out to be a more complicated magnetic state which features a triangle (or arrow) of opposite contrast to that of the background (Fig. 4); note also the presence of the three-state domain ("diamond" state); we shall discuss the energetics of these states in Sec. IV but we point out that these states have been predicted numerically. ${ }^{58,62}$

For the thinner disks, the three-domain state prevails, as can be observed in Fig. 5. The vortex state is also observed in some disks and the "triangle" state mentioned before is also present. In Table I we summarize the average number of states observed in the different polycrystalline Co disk arrays. In one instance, $t=10 \mathrm{~nm}$, we have taken two images, the second immediately after remagnetizing the sample with 


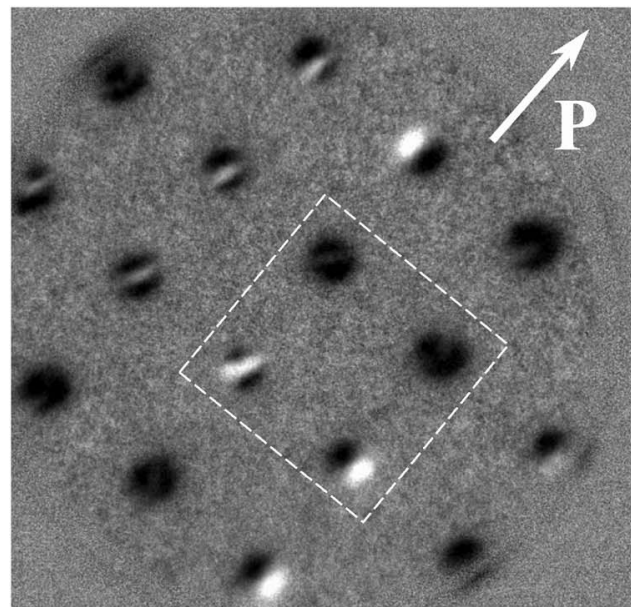

(a)

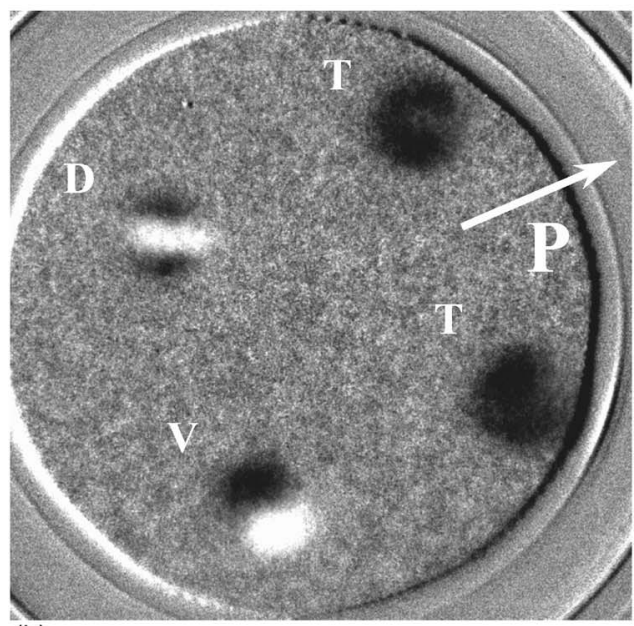

(b)

FIG. 5. PEEM images of the $10 \mathrm{~nm}$ Co polycrystalline disks in the remanent state after saturation (magnetic field applied along the direction opposite to the light polarization $\mathbf{P}$ ). The magnetic states of the disks on the high resolution image (marked on the top image) are identified as triangle $(T)$ and diamond states $(D)$.

a $4 \mathrm{kOe}$ magnetic field. It is observed that the statistics of the observed states changes dramatically, illustrating, on the one hand, the instability of the triangle state and, on the other hand, the fact that immediately after saturation, the disks tend more often to fall into the diamond state rather than the vortex state. This demonstrates the difficulty of determining

TABLE I. Thickness variation of the relative number of magnetic states (in \%) observed in polycrystalline Co disks (the total number of disks imaged is given in the last row).

\begin{tabular}{lrrrrrrr}
\hline \hline & \multicolumn{7}{c}{ thickness (nm) } \\
\cline { 2 - 8 } state & 2 & 10 & 10 & 16 & 19 & 22 & 38 \\
\hline vortex & 52 & 16 & 20 & 24 & 40 & 92 & 96 \\
diamond & 28 & 84 & 40 & 4 & 4 & 8 & 4 \\
triangle & 20 & 0 & 40 & 72 & 56 & 0 & 0 \\
disks imaged & 17 & 20 & 15 & 24 & 22 & 24 & 23 \\
\hline \hline
\end{tabular}

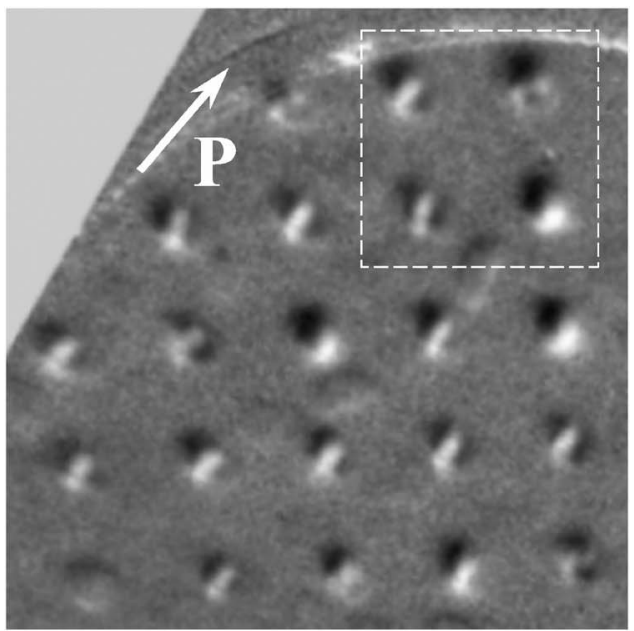

(a)

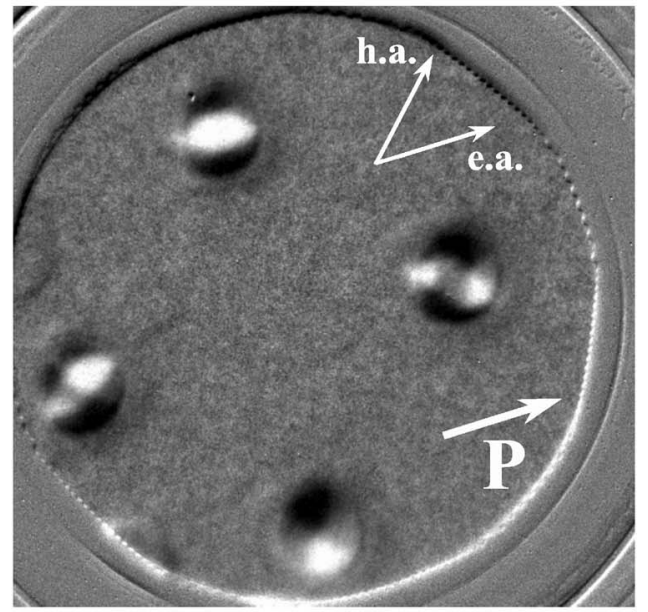

(b)

FIG. 6. PEEM images of the $29 \mathrm{~nm}$ fcc Co epitaxial disks, sample initially magnetized along the easy axis direction (along the direction of the light polarization $\mathbf{P}$ ). The bottom image corresponds to a high resolution image of the disks marked on the top image (this has been rotated by $45^{\circ}$ for convenience of display).

the relative stability of the magnetic states from the relative abundance of the different magnetic configurations alone, and also underlines the importance of using a noninvasive technique for the magnetic imaging of such states; it is likely that a small disturbance (produced by stray fields, for instance) may induce transitions between metastable magnetic states; this effect or simply thermal magnetic relaxation bringing the disks to the ground state, may be responsible for the different results of these two magnetic images.

We also imaged two fcc Co samples with thicknesses 16 and $29 \mathrm{~nm}$; we shall concentrate on the $29 \mathrm{~nm}$ Co disks, for which higher resolution images were taken. We first imaged the Co disks after the sample had been saturated along an in-plane hard magnetic axis $(\langle 100\rangle$ direction), and it is seen that most disks fall into the vortex state; when the sample was then remagnetized along a $\langle 110\rangle$ in-plane easy axis direction, a more rich variety of states are observed, as shown in Fig. 6. In the high resolution images, it is observed that in addition to the vortex state, the "diamond" state is also 


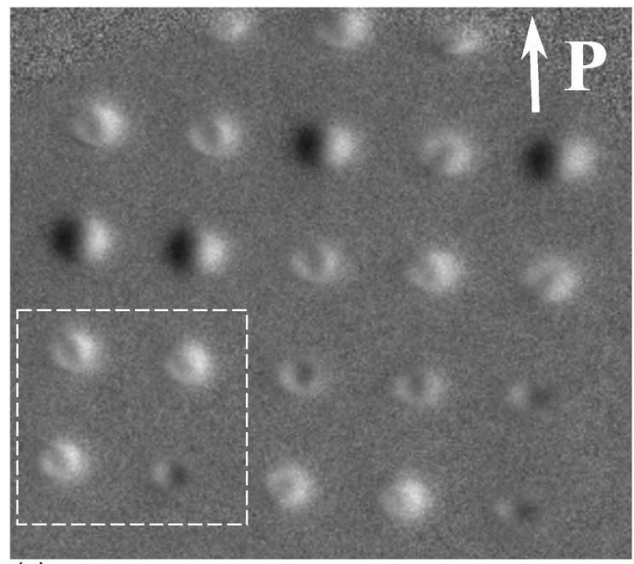

(a)

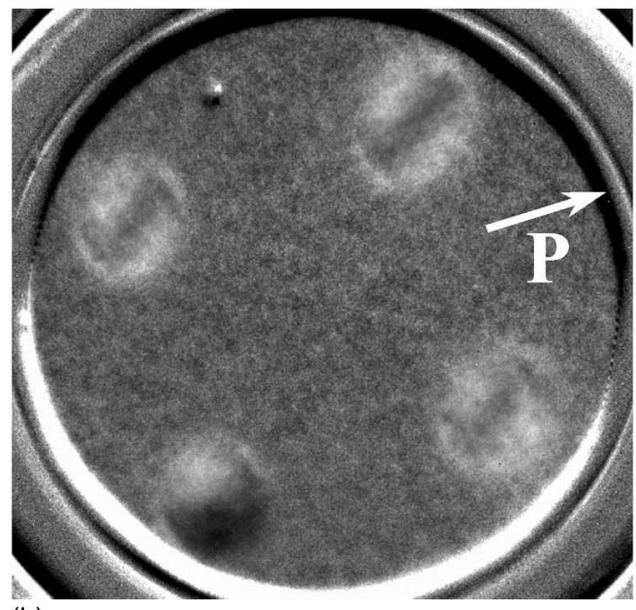

(b)

FIG. 7. PEEM images of the $10 \mathrm{~nm}$ NiFe polycrystalline disks at remanence, after saturation (magnetic field applied along the direction of the light polarization $\mathbf{P}$ ). The bottom image corresponds to a high resolution image of the disks marked on the top image.

present, with the inner domain oriented along hard and easy axis directions. It is likely that the presence of a wider pinning energy distribution of stronger pinning centers in epitaxial films compared with polycrystalline films, ${ }^{18}$ is responsible for the stabilization of the diamond state at this large Co thickness.

We mention finally the polycrystalline $\mathrm{NiFe}$ disks, for which three thicknesses were imaged, 5, 10, and $34 \mathrm{~nm}$. For the $5 \mathrm{~nm}$ disks, the PEEM image showed that all disks had relaxed into the vortex state (the $M-H$ loop for these disks showed a smaller coercivity than that of the $10 \mathrm{~nm}$ disks; in general the magnetic behavior of thinner than $\sim 3-5 \mathrm{~nm}$ $\mathrm{NiFe}$ elements seem to deviate from the trend expected from thicker films, possibly due to the influence of thermal excitations which become important in thin soft elements). The PEEM images show that all $10 \mathrm{~nm} \mathrm{NiFe} \mathrm{disks} \mathrm{fall} \mathrm{into} \mathrm{either}$ the vortex or the diamond state, Fig. 7; while some states look similar to the triangle state in the low resolution image, at higher resolution it is observed that this state is the diamond state with the inner domain rotated by $45^{\circ}$ with respect to the light polarization. The $34 \mathrm{~nm} \mathrm{NiFe} \mathrm{disks} \mathrm{have} \mathrm{all}$ fallen into the vortex state. These results are consistent with
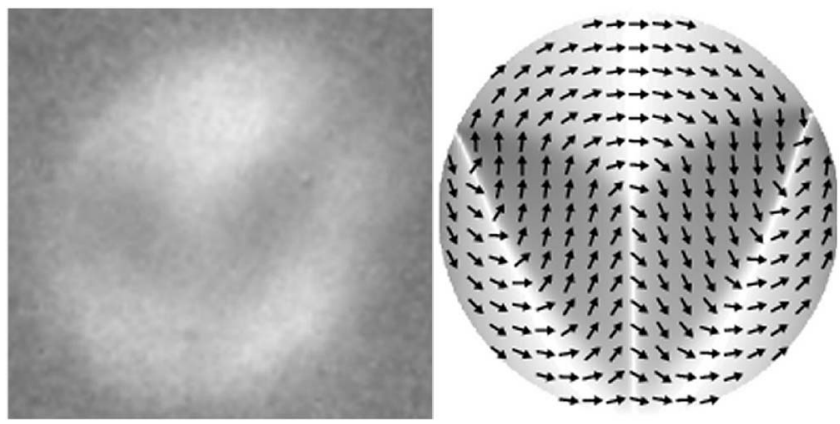

FIG. 8. PEEM image of a disk $(19 \mathrm{~nm} \mathrm{Co})$ in the triangle state (left) and corresponding micromagnetic simulation (right). The magnetic field was initially applied along the horizontal direction, and the magnetic contrast is along the same direction.

the MOKE results, which show a relatively large coercivity and remanence for the thinner $\mathrm{NiFe}$ disks, and no coercivity and zero remanence for the $34 \mathrm{~nm}$ disks.

\section{DISCUSSION}

Our experimental results show that several magnetic states are present in micrometer size disk elements, where the vortex state is expected to be the state of lowest energy. To study in more detail the energetics of these magnetic configurations and their relative stability, we performed micromagnetic simulations of disk elements using the OOMMF package. ${ }^{59}$ The parameters used for the simulations were $K_{1}=0$ for the anisotropy constant of the polycrystalline elements $K_{1}=-6.5 \times 10^{5} \mathrm{erg} / \mathrm{cm}^{3}$ for fcc Co (Refs. 13 and 60) (corresponding to cubic anisotropy with easy axis along the $\langle 111\rangle$ directions), $A=3 \times 10^{-6} \mathrm{erg} / \mathrm{cm}, M_{s}=1400 \mathrm{emu} / \mathrm{cm}^{3}$ for $C o$ and $A=1.3 \times 10^{-6} \mathrm{erg} / \mathrm{cm}, M_{s}=860 \mathrm{emu} / \mathrm{cm}^{3}$ for $\mathrm{NiFe}$. For the micromagnetic simulations we assumed the magnetization to be uniform across the out of plane direction, and a cell size of $(4 \mathrm{~nm})^{2}$ in the plane. Some states were obtained by magnetic relaxation from the uniform state, while for other states we started from a configuration close to that observed experimentally (for the larger thicknesses, some states were not stable with respect to the maximum allowed local torque); we concentrate next on the results obtained for polycrystalline $\mathrm{Co}$ and $\mathrm{NiFe}$ disks. Proceeding this way, we were able to reproduce the states imaged by PEEM (the vortex, the diamond and the triangle state) and in addition we obtained a new state which is similar to the $S$ state observed in rectangular elements, ${ }^{61}$ which we did not observe experimentally (it has been reported in micromagnetic studies of smaller NiFe circular elements ${ }^{62,63}$ ). A $C$ state has also been suggested from micromagnetic simulations, ${ }^{62-64}$ and observed for submicrometer NiFe dots ${ }^{65}$ but we did not observe this state experimentally, nor numerically; this state has a very large stray field and is not expected to be stable for micrometer sized disks. In Fig. 8 we show a high resolution PEEM image of a disk in the triangle state, which is compared with the result of a micromagnetic simulation for a disk with identical parameters. This state has a very large magnetic moment, but a relatively small stray field since the 

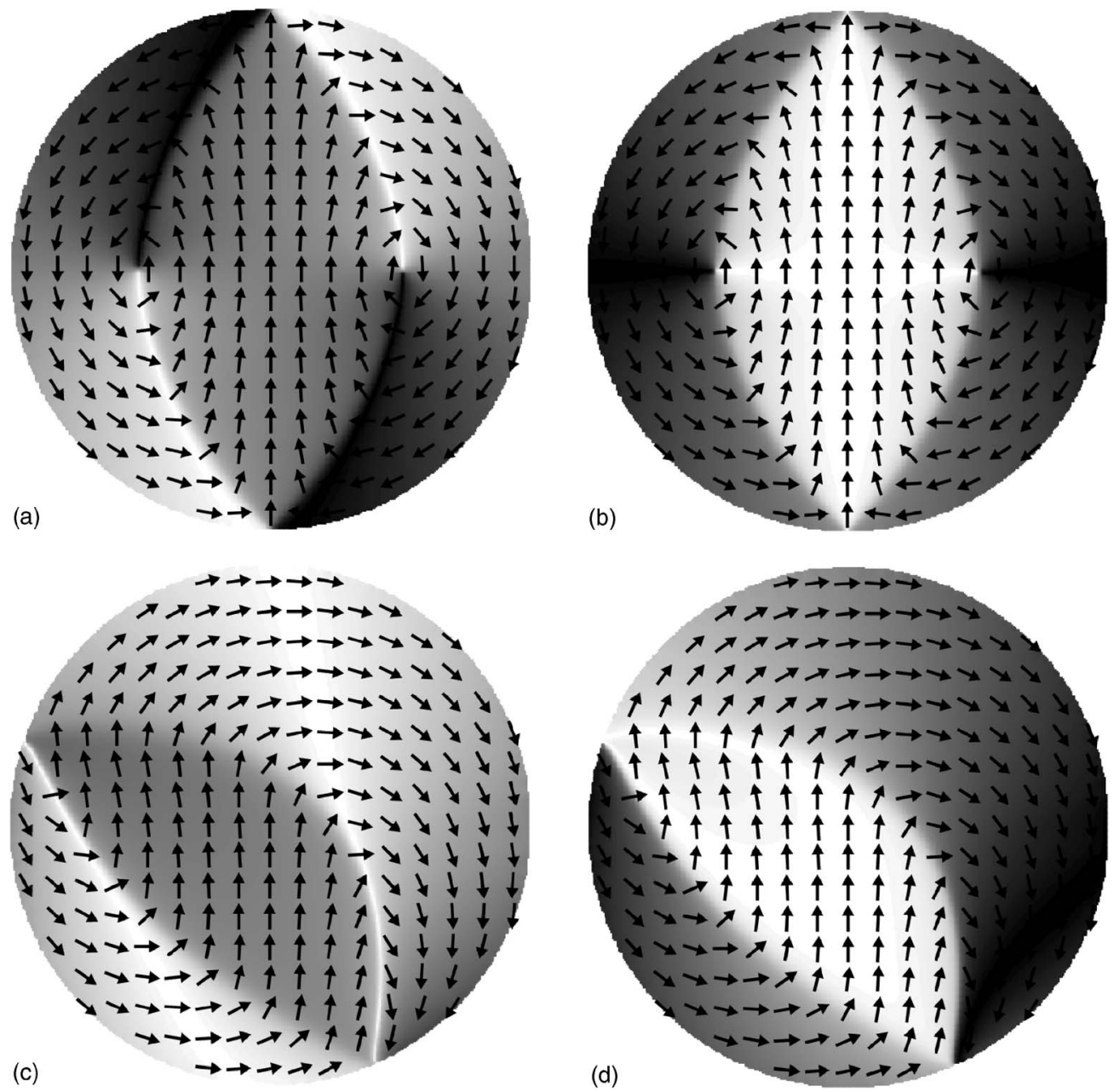

FIG. 9. Micromagnetic simulations of the diamond state (top) and of the $S$ state (bottom) for the 20 nm polycrystalline Co disk. The arrows indicate the direction of the magnetization while the color contrast refers to the horizontal (left) and vertical (right) component of the magnetization.

magnetization follows the perimeter of the disk in order to avoid the creation of "pole charges;" however, there is a significant stray field at two edges of the "triangle" which makes the magnetostatic energy the main contribution to the magnetic energy. In Fig. 9 we show the micromagnetic results corresponding to the diamond state and the $S$ state. While the vortex and the diamond state have both zero remanence, the $S$ and triangle states have large remanences; in fact, the remanence of the triangle state is very similar to the remanence observed in the $M-H$ loops of Co up to $20 \mathrm{~nm}$ in thickness, see Fig. 2. In terms of the energy components, the diamond state accommodates two vortices ${ }^{58,66}$ with a stronger twisting of the spins than that of the vortex state (leading to a comparatively larger contribution from the exchange energy term), but the largest energy component comes from the demagnetizing energy as a result of the small stray field leakage, which is also responsible for the thickness dependence (the total demagnetizing energy varies as $t^{2}$ for in-plane magnetized elements). The triangle and $S$ states have what amounts to two "edge vortices", where a large stray field leakage occurs, which makes the magnetostatic term the dominant energy contribution. Although the $S$ state has slightly lower energy than the triangle state, according to the micromagnetic simulations, this state was not observed experimentally. In Fig. 10 we plot the micromagnetic results for the thickness dependence of the energy density, normalized to the magnetostatic energy of $\mathrm{Co}, 2 \pi\left(M_{s}^{\mathrm{Co}}\right)^{2}=1.23$ $\times 10^{7} \mathrm{erg} / \mathrm{cm}^{3}$, for both the Co (symbols) and the $\mathrm{NiFe}$ (lines) disks, the latter data multiplied by a factor of 2.5. We see that the NiFe data scales relatively well to that of the Co data, with a scaling factor which is close to the ratio between the magnetostatic energies of $\mathrm{Co}$ and $\mathrm{Ni}, 2.65$. This is an indication of the dominance of the magnetostatic energy term in the total magnetic energy. It is observed that indeed the state of lowest energy is the vortex state, with almost no thickness variation, as expected since the exchange energy scales with the thickness (there is only a gentle positive slope due to edge roughness, introduced by cell discretization, which contributes to the magnetostatic energy). In order of decreasing energy density comes the triangle state, the $S$ state, the diamond state, and the vortex state, with the fluxclosed states much lower in energy. We see therefore that these different magnetic configurations do not correspond to degenerate states of the system, but rather to different energy states. One important result we can obtain from these energy estimates is the energy threshold below which metastable 


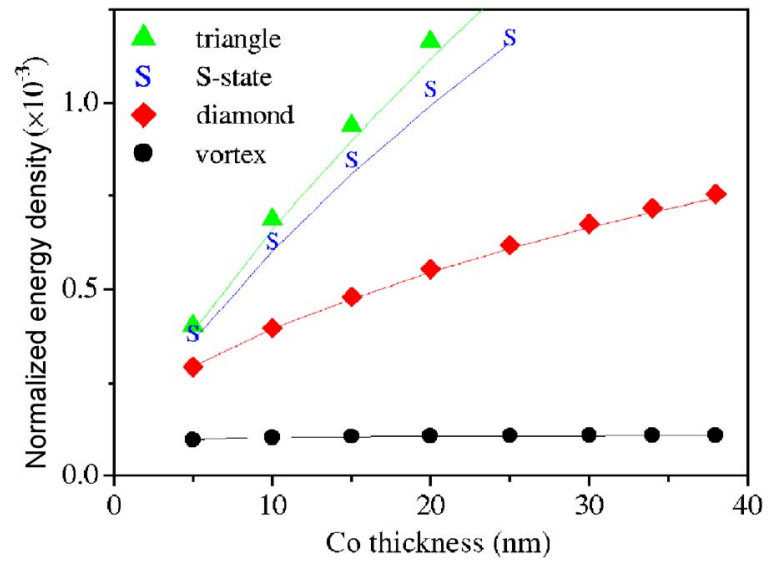

FIG. 10. (Color online) Total magnetic energy for the different magnetic states of Co disks as a function of thickness, normalized to the magnetostatic energy of $\mathrm{Co}, 2 \pi\left(M_{s}^{\mathrm{Co}}\right)^{2}$. The lines correspond to the $\mathrm{NiFe}$ data (calculated at the same thickness values as for the Co disks) multiplied by a factor of 2.5 .

states can still be stabilized; from our experimental results, we see that for Co disks $19 \mathrm{~nm}$ thick, the triangle state is still observed, while this state was not observed for the $22 \mathrm{~nm}$ disks, indicating that for the latter thicknesses the stability of this state is strongly reduced, also in agreement with the MOKE data. From Fig. 10 we may estimate the maximum energy difference between the high energy triangle state and the ground (vortex) state as $\sim 1.1 \times 10^{-3} E_{\mathrm{MS}}^{\mathrm{Co}}=1.4$ $\times 10^{4} \mathrm{erg} / \mathrm{cm}^{3}$; this energy threshold is not attained for the diamond state in the Co thickness range studied, which is consistent with the fact that this state is observed over all the thickness range considered in this study.

The stability of these metastable states, some of which have very large energy differences relative to the ground state, is attributed to pinning of the magnetization. The sources of the pinning in these metal systems range from magnetoelastic coupling of the magnetization with local strain variations at grain boundaries or at crystalline defects (misfit dislocations, interface strain or impurities); to edge and surface roughness, which may induce local magnetization configurations with a reduced magnetostatic energy; and to frustration introduced by local anisotropies from the small crystallites in the polycrystalline samples. The result, when these local potentials are larger than thermal excitations, is the stabilization of magnetic states which lie in the path of the magnetic relaxation process. The interest in the study of these metastable magnetic states is therefore twofold: on the one hand, it allows us to trace the switching of the magnetization process and on the other it draws attention to the possibility of multiple magnetic states where otherwise simple configurations would be expected. This last point has important implications for the practical implementation of small elements where well defined magnetic states and magnetic switching processes are crucial.

\section{CONCLUSIONS}

In conclusion, we have presented a systematic study of the remanent magnetic states of noninteracting $\mathrm{Co}$ and $\mathrm{NiFe}$ disk elements, as imaged by photoemission electron microscopy (PEEM). Several magnetic states were observed other than the vortex state: the diamond state, which is observed over all the thickness range studied and which has zero remanence, and the triangle state, which was observed in Co disks up to $19 \mathrm{~nm}$ in thickness and which displays a large remanence. The observation of these magnetic states explain the large coercivities in the $M-H$ curves for the thinner Co and NiFe disks. The energetics of these magnetic states was studied in the framework of the micromagnetic theory of magnetic domains; the simulations show the presence of another metastable state ( $S$ state), which was not observed experimentally and we find that the magnetic energy increases from the vortex, to the diamond, $S$, and the triangle states. The magnetostatic contribution dominates the magnetic energy, which also explains the fact that the NiFe energy values scale with those of the Co disks by a factor close to the ratio between the respective magnetostatic energies. While these states are stabilized by pinning of the magnetization (due to defects, impurities, grain boundaries, etc.), our results point to the importance of the particular switching path in stabilizing these states. This issue is likely to be critical in achieving well defined and reproducible magnetic states in small elements.

\section{ACKNOWLEDGMENTS}

This work was supported by the EPSRC (U.K.), the CMI Magnetoelectronic Devices Project, and the Gottlieb Daimler- and Karl Benz-Foundation (M.K.). Part of this work was performed at the SLS, Paul Scherrer Institute, Villigen, Switzerland.

\footnotetext{
*Present address: Fachbereich Physik, Universität Konstanz, Universitätsstr. 10, D-78457 Konstanz, Germany.

†Electronic address: jacb1@phy.cam.ac.uk

${ }^{1}$ J. Frenkel and J. Dorfman, Nature (London) 126, 274 (1930).

${ }^{2}$ L. D. Landau and E. M. Lifshitz, Phys. Z. Sowjetunion 8, 153 (1935).

${ }^{3}$ C. Kittel, Phys. Rev. 70, 965 (1946).

${ }^{4}$ C. Kittel, Rev. Mod. Phys. 21, 541 (1949).

${ }^{5}$ W. F. Brown, Jr., Micromagnetics (Interscience Publishers, New
}

York, 1963).

${ }^{6} \mathrm{~A}$. Aharoni, Introduction to the Theory of Ferromagnetism (Clarendon Press, Oxford, 1996).

${ }^{7}$ A. Hubert and R. Schäfer, Magnetic Domains (Springer-Verlag, Berlin, 1998).

${ }^{8}$ R. P. Cowburn, D. K. Koltsov, A. O. Adeyeye, M. E. Welland, and D. M. Tricker, Phys. Rev. Lett. 83, 1042 (1999).

${ }^{9}$ G. Gubbiotti, L. Albani, G. Carlotti, M. D. Crescenzi, E. D. Fabrizio, A. Gerardino, O. Donzelli, F. Nizzoli, H. Koo, and R. D. 
Gomez, J. Appl. Phys. 87, 5633 (2000).

${ }^{10}$ Y. G. Yoo, M. Kläui, C. A. F. Vaz, L. Heyderman, and J. A. C. Bland, Appl. Phys. Lett. 82, 2470 (2003).

${ }^{11}$ F. J. Castaño, C. A. Ross, C. Frandsen, A. Eilez, D. Gil, H. I. Smith, M. Redjdal, and F. B. Humphrey, Phys. Rev. B 67, 184425 (2003).

${ }^{12}$ X. Zhu, P. Grütter, V. Metlushko, Y. Hao, F. J. Castaño, C. A. Ross, B. Ilic, and H. I. Smith, J. Appl. Phys. 93, 8540 (2003).

${ }^{13}$ C. A. F. Vaz, L. Lopez-Diaz, M. Kläui, J. A. C. Bland, T. L. Monchesky, J. Unguris, and Z. Cui, Phys. Rev. B 67, 140405(R) (2003).

${ }^{14}$ M. Kläui et al., Phys. Rev. B 68, 134426 (2003).

${ }^{15}$ M. E. Schabes and H. N. Bertram, J. Appl. Phys. 64, 1347 (1988)

${ }^{16}$ R. P. Cowburn, A. O. Adeyeye, and M. E. Welland, Phys. Rev. Lett. 81, 5414 (1998).

${ }^{17}$ R. D. McMichael, J. Eicke, M. J. Donahue, and D. G. Porter, J. Appl. Phys. 87, 7058 (2000).

${ }^{18}$ C. A. F. Vaz, M. Kläui, J. A. C. Bland, L. J. Heyderman, and F. Nolting, J. Appl. Phys. 95, 6732 (2004).

${ }^{19}$ R. M. H. New, R. F. W. Pease, and R. L. White, J. Vac. Sci. Technol. B 13, 1089 (1995).

${ }^{20}$ R. M. H. New, R. F. W. Pease, and R. L. White, J. Magn. Magn. Mater. 155, 140 (1996).

${ }^{21}$ R. D. Gomez, M. C. Shih, R. M. H. New, R. F. W. Pease, and R. L. White, J. Appl. Phys. 80, 342 (1996).

${ }^{22}$ M. M. Miller, G. A. Prinz, S.-F. Cheng, and S. Bounnak, Appl. Phys. Lett. 81, 2211 (2002).

${ }^{23}$ M. Kläui, C. A. F. Vaz, J. A. C. Bland, W. Wernsdorfer, G. Faini, E. Cambril, and L. J. Heyderman, Appl. Phys. Lett. 83, 105 (2003).

${ }^{24}$ N. Dao and S. L. Whittenburg, IEEE Trans. Magn. 39, 2525 (2003).

${ }^{25}$ L. Berger, J. Appl. Phys. 55, 1954 (1984).

${ }^{26}$ P. P. Freitas and L. Berger, J. Appl. Phys. 57, 1266 (1985).

${ }^{27}$ C.-Y. Hung and L. Berger, J. Appl. Phys. 63, 4276 (1988).

${ }^{28}$ J. C. Slonczewski, J. Magn. Magn. Mater. 159, L1 (1996).

${ }^{29}$ T. Taniyama, I. Nakatani, H. Yanagihara, and E. Kita, J. Magn. Magn. Mater. 196-197, 77 (1999).

${ }^{30}$ U. Ebels, A. Radulescu, Y. Henry, L. Piraux, and K. Ounadjela, Phys. Rev. Lett. 84, 983 (2000).

${ }^{31}$ M. Kläui, C. A. F. Vaz, J. Rothman, J. A. C. Bland, W. Wernsdorfer, G. Faini, and E. Cambril, Phys. Rev. Lett. 90, 097202 (2003).

${ }^{32}$ C. A. F. Vaz, E. Blackburn, M. Kläui, J. A. C. Bland, L. Gan, W. F. Egelhoff, Jr., G. Faini, E. Cambril, and W. Wernsdorfer, J. Appl. Phys. 93, 8104 (2003).

${ }^{33}$ G. Tatara and H. Kohno, Phys. Rev. Lett. 92, 086601 (2004).

${ }^{34}$ M. Kläui, C. A. F. Vaz, J. A. C. Bland, W. Wernsdorfer, G. Faini, E. Cambril, L. J. Heyderman, F. Nolting, and U. Rüdiger, Phys. Rev. Lett. 94, 106601 (2005).

${ }^{35}$ J. Shibata, G. Tatara, and H. Kohno, Phys. Rev. Lett. 94, 076601 (2005).

${ }^{36}$ M. Kläui, P.-O. Jubert, R. Allenspach, A. Bischof, J. A. C. Bland, G. Faini, U. Rüdiger, C. A. F. Vaz, L. Vila, and C. Vouille, Phys. Rev. Lett. 95, 026601 (2005).

${ }^{37}$ L. Thomas, M. Hayashi, C. Rettner, and S. S. Parkin, 49th Annual Conference on Magnetism and Magnetic Materials (Jackson- ville, Florida, 2004).

${ }^{38}$ A. Yamaguchi, S. Nasu, H. Tanigawa, T. Ono, K. Miyake, K. Mibu, and T. Shinjo, Appl. Phys. Lett. 86, 012511 (2005).

${ }^{39}$ C. M. Schneider et al., Appl. Phys. Lett. 85, 2562 (2004).

${ }^{40}$ M. Buess, R. Höllinger, T. Haug, K. Perzlmaier, U. Krey, D. Pescia, M. R. Scheinfein, D. Weiss, and C. H. Back, Phys. Rev. Lett. 93, 077207 (2004).

${ }^{41}$ J. Raabe, C. Quitmann, C. H. Back, F. Nolting, S. Johnson, and C. Buehler, Phys. Rev. Lett. 94, 217204 (2005).

${ }^{42}$ L. J. Heyderman, M. Kläui, J. Rothman, C. A. F. Vaz, and J. A. C. Bland, J. Appl. Phys. 93, 7349 (2003).

${ }^{43}$ L. J. Heyderman, C. David, M. Kläui, C. A. F. Vaz, and J. A. C. Bland, J. Appl. Phys. 93, 10011 (2003).

${ }^{44}$ A. O. Adeyeye, J. A. C. Bland, C. Daboo, and D. G. Hasko, Phys. Rev. B 56, 3265 (1997).

${ }^{45}$ C. Mathieu et al., Appl. Phys. Lett. 70, 2912 (1997).

${ }^{46}$ M. Kläui, C. A. F. Vaz, J. A. C. Bland, and L. J. Heyderman, Appl. Phys. Lett. 86, 032504 (2005).

${ }^{47}$ C.-A. Chang, Appl. Phys. Lett. 57, 297 (1990).

${ }^{48}$ R. Naik, C. Kota, J. S. Payson, and G. L. Dunifer, Phys. Rev. B 48, 1008 (1993).

${ }^{49}$ B. G. Demczyk, V. M. Naik, A. Lukaszew, R. Naik, and G. W. Auner, J. Appl. Phys. 80, 5035 (1996).

${ }^{50}$ C. A. F. Vaz and J. A. C. Bland, Phys. Rev. B 61, 3098 (2000).

${ }^{51}$ M. Kläui, C. A. F. Vaz, L. Lopez-Diaz, and J. A. C. Bland, J. Phys.: Condens. Matter 15, R985 (2003).

${ }^{52}$ C. M. Schneider, R. Frömter, C. Ziethen, W. Swiech, N. B. Brookes, G. Schönhense, and J. Kirschner, in Magnetic Ultrathin Films, Multilayers and Surfaces-1997, edited by D. D. Chambliss et al., MRS Symp. Proc. No. 475 (Materials Research Society, Warrendale, 1997), p. 381.

${ }^{53}$ G. Schönhense, J. Phys.: Condens. Matter 11, 9517 (1999).

${ }^{54}$ A. Locatelli, K. S. Cherifi, S. Heun, M. Marsi, K. Ono, A. Pavlovska, and E. Bauer, Surf. Rev. Lett. 9, 171 (2002).

${ }^{55}$ A. Scholl, H. Ohldag, F. Nolting, J. Stöhr, and H. A. Padmore, Rev. Sci. Instrum. 73, 1362 (2002).

${ }^{56}$ Nanoscience and Nanotechnology, edited by H. Hopster and H. P. Oepen (Springer-Verlag, Berlin, 2005).

${ }^{57}$ A. Fernandez and C. J. Cerjan, J. Appl. Phys. 87, 1395 (2000).

${ }^{58}$ J.-G. Zhu and Y. Zheng, in Spin Dynamics in Confined Magnetic Structures I, edited by B. Hillebrands and K. Ounadjiela (Springer-Verlag, Berlin, 2002), p. 289.

${ }^{59}$ http://math.nist.gov/oommf.

${ }^{60}$ J. Rothman, M. Kläui, L. Lopez-Diaz, C. A. F. Vaz, A. Bleloch, J. A. C. Bland, Z. Cui, and R. Speaks, Phys. Rev. Lett. 86, 1098 (2001).

${ }^{61}$ Y. Zheng and J.-G. Zhu, J. Appl. Phys. 81, 5471 (1997).

${ }^{62}$ K. Yu. Guslienko, V. Novosad, Y. Otani, H. Shima, and K. Fukamichi, Phys. Rev. B 65, 024414 (2002).

${ }^{63}$ J. K. Ha, R. Hertel, and J. Kirschner, Phys. Rev. B 67, 224432 (2003).

${ }^{64}$ Z.-H. Wei, M.-F. Lai, C.-R. Chang, N. A. Usov, J. C. Wu, and J.-Y. Lai, IEEE Trans. Magn. 39, 2675 (2003); J. Magn. Magn. Mater. 282, 11 (2004).

${ }^{65}$ M. Heumann, T. Uhlig, and J. Zweck, Phys. Rev. Lett. 94, 077202 (2005)

${ }^{66}$ I. L. Prejbeanu, M. Natali, L. D. Buda, U. Ebels, A. Lebib, Y. Chen, and K. Ounadjela, J. Appl. Phys. 91, 7343 (2002). 